\title{
Meningoencefalitis Zigomicótica Piogranulomatosa en un Puercoespín de Cola Prensil (Coendou Prehensilis)
}

\author{
Piogranulomatous Zygomycotic Meningoencephalitis \\ in a Prehensile Tailed Porcupine (Coendou Prehensilis)
}

"Ciuoderis-Aponte, K. A. \& ** Ochoa-Amaya, J. E.

CIUODERIS-APONTE, K. A. \& OCHOA-AMAYA, J. E. Meningoencefalitis zigomicotica piogranulomatosa en un puercoespin de cola prensil (Coendou Prehensilis). Int. J. Morphol., 27(4):1187-1194, 2009.

RESUMEN: Con el término de zigomicosis (ZM) se designa un grupo de enfermedades causadas por hongos de la clase Zygomicetes, de los órdenes Mucorales y Entomophthorales, de varios géneros y especies. La presentación de ZM en animales ocurre esporádicamente en numerosas especies tanto en animales domésticos como silvestres (incluso en mamíferos marinos) y son pocos los casos comprobados de aislamiento e identificación del agente causal. Pocos hongos son considerados como neurotrópicos ya que tienden a localizarse en el SNC después de invadir el hospedador; sin embargo, la mayoría de los casos de meningitis o meningoencefalomielitis son parte de micosis sistémicas como la Candidiasis, la Criptococosis, la Aspergilosis o la ZM. El presente trabajo documenta el hallazgo de una meningoencefalitis zigomicotica secundaria a un trauma craneoencefálico en un puercoespín de cola prensil ( Coendou prehensilis), siendo una enfermedad rara infrecuente en el sistema nervioso central tanto en humanos como en animales y adicionalmente sin reportes previos en esta especie.

PALABRAS CLAVE: Sistema nervioso central; Infección fúngica; Zigomicosis; Puercoespín.

\section{INTRODUCCIÓN}

La histopatología permite evidenciar la morfología de los hongos y evaluar su relación con las lesiones tisulares, lo que representa una información valiosa para el diagnóstico de micosis en patología veterinaria (Pérez \& Carrasco, 2000). Las enfermedades micóticas tienen importancia en patología veterinaria debido al carácter zoonósico de la mayoría de estos procesos, a las pérdidas que provocan en animales de producción y a que pueden afectar a especies silvestres (Pérez \& Carrasco).

Debido a que la mayoría de los hongos potencialmente patógenos para el hombre y los animales son saprofitos, su aislamiento desde una lesión, no implica necesariamente que sean los responsables del proceso patológico (Barker et al., 1993; Scott et al., 1995), sino que debe de acompañarse de un estudio histopatológico que permita evidenciar la morfología de los elementos micóticos y su relación con las lesiones tisulares, que en ocasiones presentan un patrón típico para algunas especies (Chandler \& Watts, 1987).
Hay dos grandes grupos de hongos en los procesos micóticos, los saprofitos y los patógenos; se considera a un hongo como patógeno cuando produce daño tisular, pero ninguna lesión histopatológica es específica de una micosis, pudiéndose hallar un variado espectro de lesiones inflamatorias (Mayayo).

Comúnmente se puede observar a los hongos con dos morfologías básicas, con una disposición tubular o hifas (multicelular) y una segunda de forma redondeada u oval que correspondería con las esporas o conidios (unicelular). Estas formas, a su vez, pueden ser pigmentadas o no. Conocidos los cambios tisulares que se producen en los tejidos y las diferentes morfologías, podemos pasar a clasificarlos, en relación a estas características, se pueden identificar y diagnosticar infecciones por hongos pertenecientes a alguno de estos cuatro grupos: dimorfos, patógenos clásicos, patógenos oportunistas emergentes y otros (Mayayo, 2004), de la misma forma su clasificación puede atender a la localización orgánica

\footnotetext{
* Medicina de la Conservación, Escuela de Medicina Veterinaria y Zootecnia, Facultad de Ciencias Agropecuarias y Recursos Naturales, Universidad de los Llanos, Colombia.

** Patología Animal, Escuela de Medicina Veterinaria y Zootecnia, Facultad de Ciencias Agropecuarias y Recursos Naturales, Universidad de los Llanos, Colombia.
} 
y a la acción patógena del hongo: dermatomicosis, micosis sistémicas y micotoxicosis (Kitt \& Schulz, 1985).

Con el término de zigomicosis (ZM) se designa un grupo de enfermedades causadas por hongos de la clase Zygomicetes, de los órdenes Mucorales y Entomophthorales, de varios géneros y especies (Acha \& Szyfres, 2001). Todos los zigomicetos se caracterizan por presentar rasgos morfológicos típicos como son hifas de paredes irregulares, de grosor variable, ocasionalmente septadas e irregularmente ramificadas (Berenguer y Rodríguez, 1998; Pérez \& Carrasco; Jubb et al., 2007), pigmentadas (Shelly, 2003) y normalmente en ángulo de $90^{\circ}$ (Mayayo).

Las técnicas de tinción rutinaria utilizadas en histopatología como la Hematoxilina \& Eosina (H\&E) no permiten evidenciar la mayoría de las especies de hongos potencialmente patógenos por lo que son necesarias técnicas de tinción especiales como la del ácido peryódico de Schiff PAS (Pérez \& Carrasco), sin embargo, el protoplasma basofílico granular de los hongos Zygomicetes puede ser visible con H\&E (Mayayo; Jubb et al.) y poco visible con la tinción PAS, pero los organismos son usualmente visualizados con la tinción Plata Metamina de Gomori (Shelly, 2003; Jubb et al.).

La presentación de ZM en animales ocurre esporádicamente en numerosas especies tanto en animales domésticos como silvestres (incluso en mamíferos marinos) y son pocos los casos comprobados de aislamiento e identificación del agente causal (Acha \& Szyfres). Aunque se ha descrito la infección por zigomicetos en todas las especies domésticas, son quizás los rumiantes los más frecuentemente afectados (Pérez \& Carrasco). La ZM puede llegar a invadir la base del cráneo y afectar el sistema nervioso central (SNC), sin embargo, cualquier localización es posible, incluso la gastrointestinal (Mayayo).

La mayoría de los patógenos zigomicetos se clasificó inicialmente como miembros del género Mucor, estos organismos fueron posteriormente reasignados, y siguen siendo reasignados, en diferentes géneros y familias dentro del orden Mucorales (Ribes et al., 2001). Incluso con la demostración de pobres resultados de cultivo, pronto se hizo evidente que Rhizopus spp. y Mucor spp. fueron los organismos predominantes que causaban la enfermedad (Ribes et al.). Otra información importante también ha sido colectada por clínicos e investigadores quienes asocian la ZM con cáncer (Baker, 1962), el uso antibióticos o de prednisona (Torack, 1957), y formas asociadas a terapias inmunosupresivas (Ribes et al.).

Las ZM producidas por hongos del orden Mucorales (géneros Absidia, Mucor, Rhizopus, entre otros) están ca- racterizadas por alta afinidad de las hifas para invadir los vasos sanguíneos (Carrasco et al., 1993; 1997; Mayayo; Jubb et al.), lo que provoca una diseminación hematógena desde la lesión inicial (Munday et al., 2006), desarrollando trombosis e infartos hemorrágicos (Kitt \& Schulz).

La infección fúngica del sistema nervioso central (SNC) es una enfermedad que pone en peligro la vida y una variedad de hongos causa la infección bien sea meningitis aguda o crónica, o la lesión por la ocupación del espacio tisular. Aunque la meningitis es la presentación más común de la micosis del SNC, algunos de los hongos filamentosos causan absceso cerebral o granuloma más comúnmente que meningitis. El SNC está protegido por una barrera mecánica frente a la colonización o invasión de hongos, sin embargo, en condiciones adecuadas los hongos pueden llegar a la SNC por vía hematógena o cuando la barrera anatómica es alterada por traumas o cirugías. El desarrollo de meningitis por hongos requiere tanto la exposición al agente patógeno como algunas deficiencias en las defensas del hospedador, permitiendo así el acceso y replicación del mismo en las meninges (Chakrabarti, 2007).

Pocos hongos son considerados como neurotrópicos ya que tienden a localizarse en el SNC después de invadir el hospedador, causando granulomas pigmentados principalmente en animales de compañía de regiones tropicales (Miller, 2008), sin embargo, la mayoría de los casos de meningitis o meningoencefalomielitis son parte de las micosis sistémicas como la Candidiasis, la Criptococosis, la Aspergilosis o la Zigomicosis (Chakrabarti).

La Zigomicosis del SNC se debe a especies de los géneros Rhizopus, Rhizomucor, Absidia, Mucor, Cunninghamella, Apophysomyces y Saksenaea, con reservorios ambientales y mecanismos de transmisión similares a los de Aspergillus spp (Sugar, 1992). Los Zigomicetos se basan en un huésped previamente, estos hongos prosperan en un ambiente muy ácido, que es rico en hidratos de carbono, así, un paciente con cetoacidosis tiene una doble amenaza porque la función fagocítica es defectuosa (debido a la acidosis) y proporciona el entorno para la rápida invasión (Chakrabarti et al., 2006; Munday et al.). La piel como puerta de entrada también ha sido descrita, en particular relacionada con vendas quirúrgicas elásticas, algunos pacientes pueden desarrollar ZM con defectos inmunológicos subyacentes (Chakrabarti et al.). La ZM aislada de cerebro ha sido también descrita (Verma et al., 2006).

El objetivo del presente trabajo es documentar el hallazgo de una meningoencefalitis Zigomicótica secundaria a un trauma craneoencefálico en un puercoespín de cola prensil (Coendou prehensilis), siendo una enfermedad rara in- 
frecuente en el sistema nervioso central tanto en humanos como en animales y adicionalmente sin reportes previos en la especie.

\section{RELATO DEL CASO}

Descripción de la especie. El Puercoespín de cola prensil (Coendou prehensilis) es una de las diez especies de roedores de la familia Erethizontidae del geotrópico (Roberts et al., 1985) o puercoespines de América. Ampliamente distribuido en bosques secos y húmedos de Colombia, el sur de Venezuela hasta el norte de Argentina (Eisenberg \& Redford, 1999; IUCN, 2008). El rango actitudinal va desde 150 hasta 2500 m.s.n.m. (Spraker). La longitud de su cuerpo llega hasta $55 \mathrm{~cm}$, la de su cola es de 33 a $48 \mathrm{~cm}$ y su peso llega hasta 4 $\mathrm{Kg}$. La parte dorsal del cuerpo está cubierta de espinas fuertes y juntas, muchas de ellas son tricolor con bandas casi de igual de ancho, blanco y amarillo pálido en la base y en la punta, negras o castaño oscuro en el centro (Spraker). Las espinas de la cabeza, patas y cola son cortas, las de la espalda son largas y gruesas; La cabeza es redonda; la nariz y los labios son grandes, bulbosos, suaves y rosados; las orejas son pequeñas; las vibrisas son muy largas, llegando hasta los hombros; la cola carece de espinas, es fuerte y prensil, con la parte superior adaptada para agarrarse a las ramas. Los pies poseen almohadillas anchas expandidas en oposición a cuatro garras, fuertes y dobladas hacia adentro. Es de hábitos nocturnos, vive en las ramas de los árboles de las que casi nunca baja, puede ser solitario o convivir en parejas, de día duerme entre los árboles huecos y prefiere los niveles altos y medios del bosque (Spraker; Nowak, 1999). Se alimenta de hojas, flores, tallos, raíces, y la corteza de algunos árboles (Roberts et al.). La gestación dura 203 días (Eisenberg \& Redford; Spraker), después de lo cual nace generalmente una sola cría cubierta de pelos rojos y pequeñas espinas que se endurecen inmediatamente después del parto. El destete ocurre hacia las 10 semanas. La madurez sexual la alcanzan en 19 meses (Eisenberg \& Redford; Spraker). Según la lista roja de especies amenazadas de la IUCN se encuentra en la categoría de Preocupación menor (LC).

Clasificación zoológica: Reino: Animalia; Filum: Chordata; Clase: Mammalia; Orden: Rodentia; Suborden: Hystricognathi; Familia: Erethizontidae; Género: Coendou; Especie: C. prehensilis - Linnaeus, 1758 (Spraker; Wilson \& Reeder, 2005).

Necropsia y hallazgos postmortem. Al consultorio de la clínica Veterinaria de la Universidad de los Llanos (Villavicencio, Colombia) fue llevado un puercoespín de cola prensil de la especie Coendou prehensilis, hembra, edad adulta, con un de peso $2 \mathrm{Kg}$. El paciente tenía procedencia de vida silvestre, se reporta rescatado en una carretera luego de un posible accidente con un automóvil y el motivo de consulta fue: Depresión, letargia, trauma craneoencefálico múltiple y traumas en miembro derecho anterior y posterior, concomitante con un politraumatismo debido a un accidente automovilístico. Los hallazgos significativos al examen clínico fueron: Hemiparesia derecha, ausencia al reflejo de amenaza, herida traumática con posible fractura en la región temporal y frontal del cráneo y epistaxis bilateral. La fractura a nivel temporal es confirmada por radiografía. Aunque el animal ingresa a la unidad de cuidados intensivos con un tratamiento apropiado para el trauma craneoencefálico, el cuadro se agravó y evolucionó pocas semanas después hacia la muerte.

El procedimiento de necropsia se realizó inmediatamente al fallecimiento del animal. Se tomaron las muestras pertinentes a los hallazgos encontrados, las cuales se fijaron en formalina Buffer al $10 \%$ y fueron enviadas al laboratorio de histopatología de la Universidad de los Llanos, posteriormente se incluyeron en parafina mediante métodos de rutina para microscopía óptica. Se realizaron cortes de 3-4 $\mathrm{mm}$ de grosor que fueron teñidos con las técnicas de Hematoxilina-Eosina (H\&E) con el siguiente reporte de necropsia: Los principales hallazgos postmortem encontrados a la inspección externa fueron: hemorragias en cavidad nasal, fractura frontal y temporal del cráneo, de las cuales sólo se encontraba en tratamiento la temporal (evidencia de síntesis quirúrgica).

Los principales hallazgos postmortem encontrados a la inspección interna fueron: en cavidad torácica se encontró la traquea, la pleura y los pulmones hemorrágicos, dilatación cardiaca derecha e hipertrofia ventricular izquierda. En cavidad abdominal se encontró el hígado con áreas blanquecinas en el lóbulo derecho y la mucosa intestinal congestionada, en cavidad pélvica los riñones se hallaron congestionados. En la cavidad craneana en las áreas de fractura se encontró necrosis y contenido purulento en el espacio epidural, hematomas múltiples y contusión cerebral y cerebelar, diagnosticándose como una leptomeningitis a nivel macroscópico.

Histopatología. El diagnóstico histopatológico se enfatizó en el sistema nervioso central, con el hallazgo microscópico de una meningoencefalitis micótica supurativa en cerebro y cerebelo. Se observó la presencia de hifas basofílicas irregulares, anchas, de pared delgada, algunas septadas, con ramificaciones irregulares, embolizando vasos sanguíneos, hay una vasculitis severa generalizada con trombos ocasionales y necrosis fibrinoide de las paredes del vaso, acompa- 


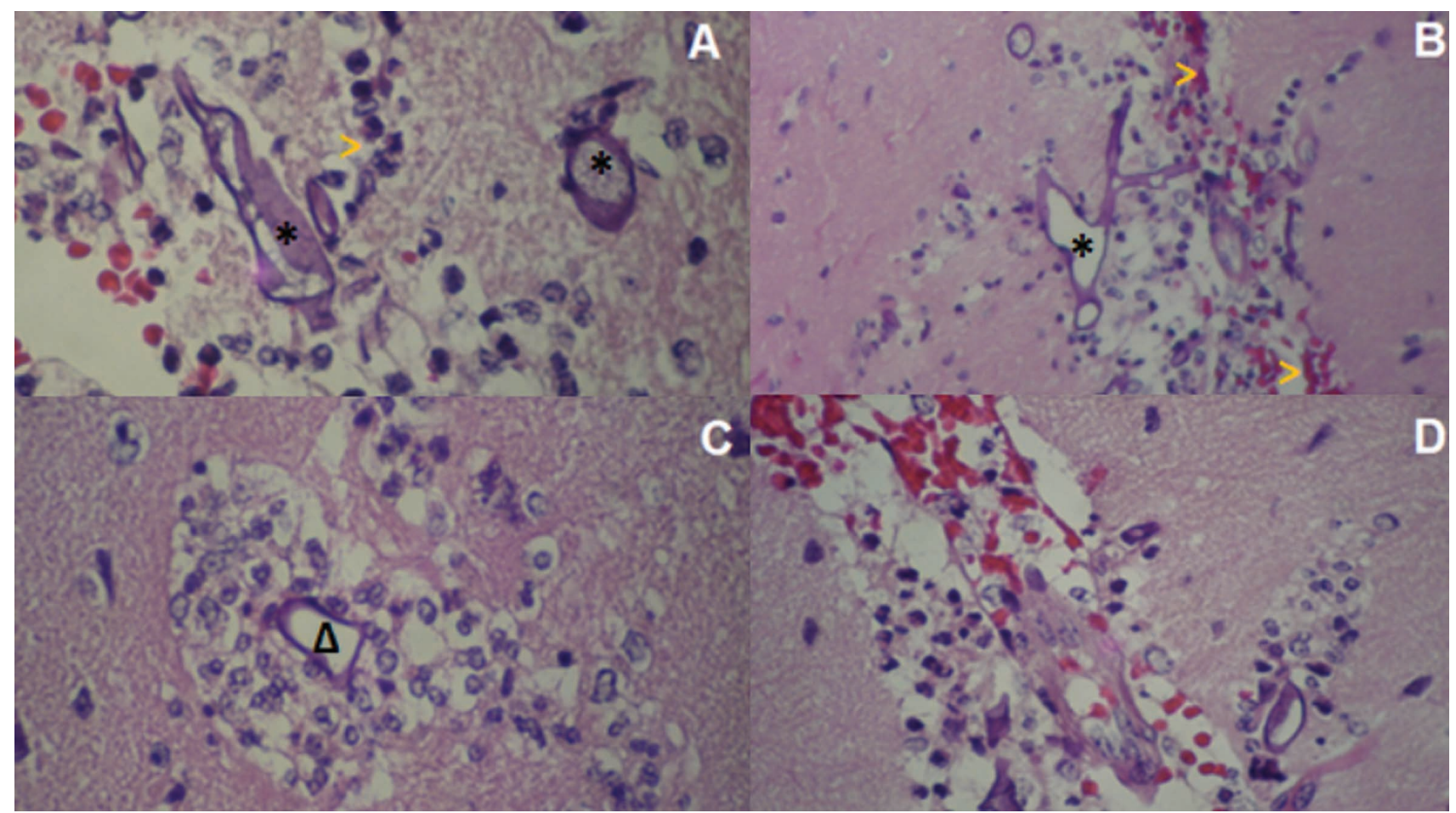

Fig. 1. Cerebro (H\&E). Hifas de paredes irregulares, de grosor variable, con forma de bola y con las dilataciones bulbosas focales [*], con infiltrado linfocitario y de PLMNs [>], 40X, (A). Organismos con formas de bolas, no septados, no dicotomos ramificados, con bifurcación irregular [*] y una reacción inflamatoria mixta con pequeñas hemorragias [>], 10X, (B). Infiltración de células histiocitarias alrededor de una hifa $[\Delta], 40 \mathrm{X},(\mathrm{C})$. Hifa de Zigomiceto, colapsada y plegada que se tuerce y semeja una cinta, (D).

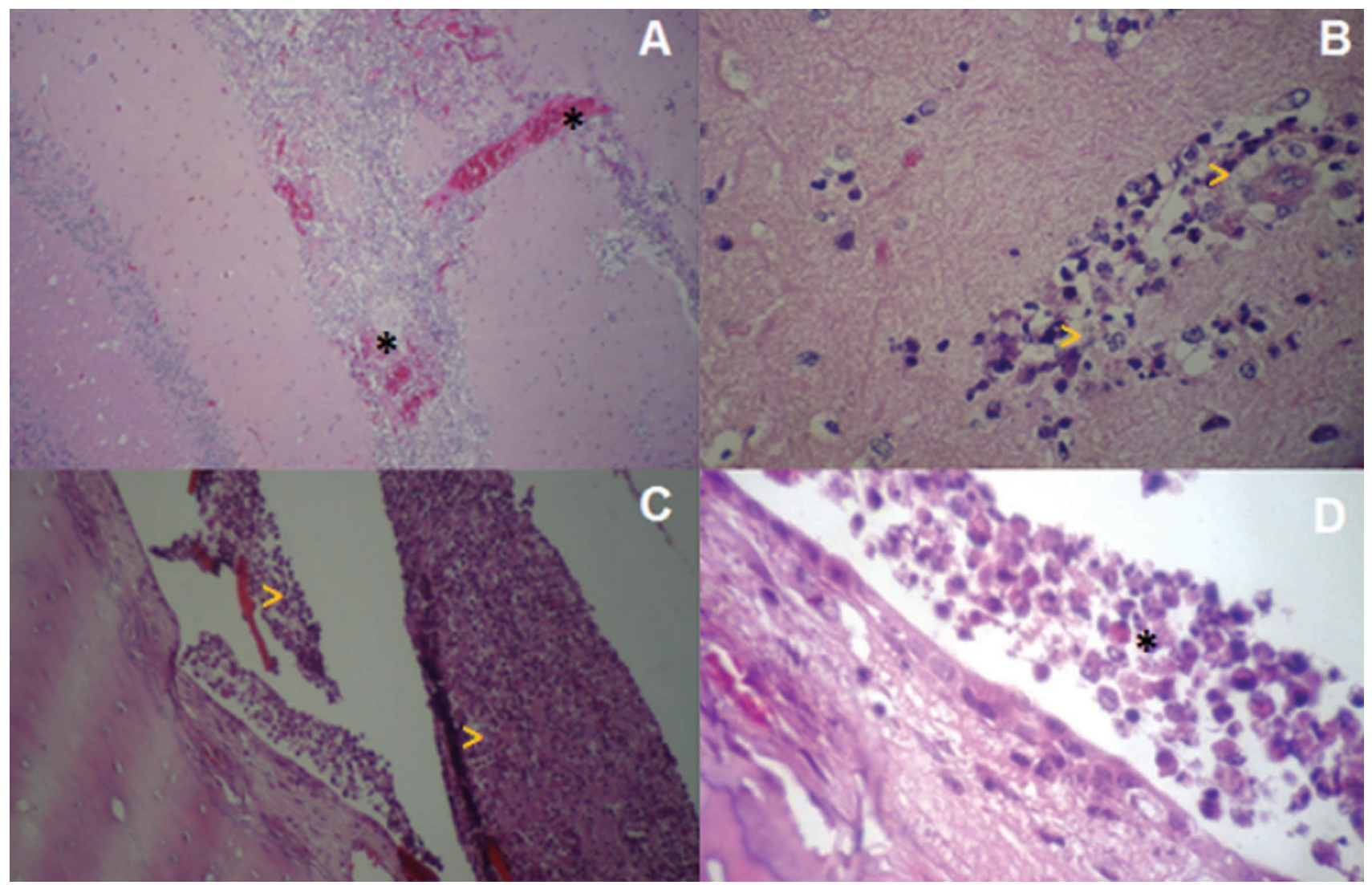

Fig. 2. Cerebelo (H\&E). Vasculitis necrotizante tromboembólica, infiltrados perivasculares principalmente de células inflamatorias [*] comprendidas de macrófagos con números menores de neutrófilos. Hay áreas localmente extensas de necrosis, edema y deposición de fibrina en los vasos sanguíneos circundantes [*], 10X (A). Edema e infiltrado de PLMNs alrededor de un vaso [>], 40X (B). Hueso temporal con fuerte infiltrado de PLMNs en el espacio subaracnoideo [>], 10X, (C). Acercamiento del campo anterior, donde se aprecian los neutrófilos [*], 40X, (D). 
ñadas de vasculitis necrotizante fibrinoide, además de focos numerosos y esparcidos aleatoriamente de necrosis licuefactiva con una fuerte reacción piogranulomatosa y una moderada microgliosis. Rodeando los vasos hay infiltrados perivasculares principalmente de células inflamatorias comprendidas de macrófagos con menores números de neutrófilos. Hay áreas localmente extensas de necrosis, edema y deposición del fibrina en los vasos sanguíneos circundantes y organismos no septados, no dicotomos ramificados (Zygomicetos) los cuales también son visibles dentro de los vasos y trombos (véase Figs. 1 y 2).

\section{DISCUSIÓN}

Los hongos de la división Zygomicota de interés en medicina veterinaria pertenecen a dos órdenes: el orden Entomoftorales y el orden Mucolares (incluyen los géneros Rhizopus, Mucor Rhizomucor, Absidia, Mortierella, entre otros), tienen las siguientes características comunes: cosmopolitas (Acha \& Szyfres) y ampliamente distribuidos en la naturaleza, saprofitos del alimento, el suelo o el aire (Vadillo et al., 2002; Jubb et al.) y hacen parte de la flora normal de la piel y el pelo (Jubb et al.), pero en determinadas condiciones debilitantes del hospedador pueden actuar como saprófitos oportunistas colonizando tejidos superficiales y provocando infecciones en la capa subcutánea de la piel, aprovechando normalmente las heridas (Jubb et al.) o através de implantación traumática (Shelly), pudiendo también colonizar órganos internos (Vadillo et al.), también se cree que la mayoría de los casos se deben a la contaminación de heridas penetrantes (Harvey \& McKeever, 2001; Medleau \& Hnilica, 2007). Son reportados como contaminantes de laboratorios (Jubb et al.) y no se conoce muy bien su papel como un patógeno nosocomial o intrahospitalario.

Las zigomicosis causadas por hongos del orden Mucorales también son llamadas mucormicosis (Berenguer \& Rodríguez; Jubb et al.), en particular por los géneros Rhizopus, Rhizomucor y Absidia, aunque otros géneros como Mucor, Cunninghamella, Mortierella, Saksenaea, Apophysomyces, Syncephalastrum, y Cokeromyces producen infecciones de forma anecdótica (Berenguer \& Rodríguez). Los principales factores predisponentes para la mucormicosis son la hiperglicemia, la acidosis metabólica, el tratamiento con glucocorticoides y la granulocitopenia (Berenguer \& Rodríguez; Jubb et al.). El SNC puede afectarse por contigüidad en formas rinocerebrales, por traumas o heridas locales o por vía hematógena en infecciones diseminadas, causando lesiones como infartos, hemorragias o abscesos cerebrales (Berenguer \& Rodríguez; Riet-Correa et al., 2008).
En este reporte de caso, el diagnóstico histopatológico se realizó a partir de muestras fijadas en formalina buferada al $10 \%$ y como en muchos casos no se sospechaba de un proceso micótico en el animal, por lo que cuando se observaron los hongos en el SNC ya no existía la posibilidad de realizar un cultivo, motivo que también describen Pérez \& Carrasco. El diagnóstico de meningoencefalitis Zigomicótica se basó en los hallazgos microscópicos y en las características morfológicas del microorganismo presente en las lesiones del sistema nervioso central, donde los tejidos evaluados microscópicamente con H\&E, presentaron lesiones necróticas licuefactivas y hemorrágicas con abundante infiltración eosinofílica, mostrando hifas irregulares ramificadas, septadas o no, reportes descritos también para casos de ZM cerebral en humanos (Sundaram et al., 2005), en bovinos (Munday et al.), en bisonte americano y venado, y en ovejas (Riet-Correa et al.).

Como ya se ha comentado, para que se produzca una lesión micótica deben existir una serie de factores que la favorezcan, como son la inmunidad del huésped, la virulencia, la cantidad de células fúngicas y la coexistencia de otras enfermedades, donde la primera respuesta ante una micosis es una inflamación supurativa con infiltrado de polimorfonucleares, pasando posteriormente a una respuesta de células mononucleares y, si evoluciona, esta presenta macrófagos, células epitelioides y células gigantes multinucleadas, dando lugar a una infección crónica y a fibrosis, sin embargo, todo esto puede estar atenuado si en el paciente coexiste una enfermedad inmunosupresora (Kumar et al., 2004), para el caso del puercoespín se evidenció una respuesta inflamatoria en el sistema nervioso central pero no concomitante con un proceso crónico, puesto que el infiltrado celular en su gran mayoría era neutrófilos, eosinófilos, linfocitos y células plasmáticas, con escasos histiocitos, posiblemente debido a una inmunosupresión o debido a una etapa inicial de la invasión.

El estrés prolongado en animales puede resultar en varios desórdenes como la alteración del comportamiento, problemas cardiovasculares, hipertensión, disminución de la conversión alimenticia, ulceración gastrointestinal, falla reproductiva, imbalance electrolítico o como pudo suceder en el caso presente una deficiencia inmunológica o inmunosupresión (Fowler, 1993). Es muy importante valorar el potencial inmunológico del paciente, que en muchos casos suele estar disminuido debido a que las micosis son mucho más frecuentes en pacientes inmunodeprimidos, es por esto que el déficit de defensas condiciona el grado de respuesta del organismo ante estos agentes patógenos (Mayayo).

Ya que los mucolares dependen del hierro, muchos aspectos de su patogenia se relacionan con la disposición de 
este catión, como por ejemplo la cetoacedosis, que reduce la capacidad de fijación del hierro quedando a libre disponibilidad del hongo y estimulando el crecimiento de estos hongos en el interior de los vasos sanguíneos (García-Rodríguez \& Picazo, 1999).

Todos los miembros de los Zygomicetos son morfológicamente similares en los tejidos y consecuentemente el cultivo es necesario para la identificación específica, aunque técnicas inmunohistoquímicas para el hongo en secciones del tejido han sido reportadas, el antisuero específico no se encuentra disponible fácilmente (Jubb et al.).

En el diagnóstico diferencial de las zigomicosis hay que incluir a otros hongos miceliares (Medleau \& Hnilica) tales como Aspergillus spp., de los que se diferencian por la morfología de las hifas; sin embargo, no es posible diferenciar la hifas de zigomicetos de las de Phytium spp., que provoca lesiones cutáneas o digestivas en regiones endémicas de climas tropicales (Scott et al.).

Para el caso del Puerco espín se asume que las lesiones hemorrágicas difusas en el cerebro y cerebelo son el resultado del trauma craneoencefálico padecido por el animal y evidenciado macroscópicamente en la región temporal y frontal. La contusión resultó en una lesión mayor e implicó hemorragias en el espacio subaracnoideo e intracerebral, estas lesiones también se acompañan de hemorragias producidas por contragolpe que son más severas y se desarrollan fuera del punto de impacto cuando el choque del cerebro contra el cráneo es muy súbito o cuando este se desplaza sobre las prominencias óseas correspondiendo con el reporte de Miller, de igual forma se observó epistaxis posiblemente resultado del mismo trauma.

\section{AGRADECIMIENTOS}

Agradecemos a la Clínica Veterinaria, a la Señorita Andrea Hernández, técnica del Laboratorio de Histopatología de la Facultad de Ciencias Agropecuarias y Recursos Naturales y a los estudiantes de noveno semestre de Medicina Veterinaria y Zootecnia del primer ciclo de 2008 de la Universidad de los Llanos, Colombia.

CIUODERIS-APONTE, K. A. \& OCHOA-AMAYA, J. E. Piogranulomatous zygomycotic meningoencephalitis in a prehensile tailed porcupine (Coendou Prehensilis). Int. J. Morphol., 27(4):1187-1194, 2009.

SUMMARY: With the word Zygomycosis (ZM) are designating a group of diseases caused by fungi of the class Zygomicetes, the orders Mucorales and Entomophthorales, of several genera and species. The presentation of ZM in animals occurs sporadically in many species both in domestic and wild animals (including marine mammals), and are checked few cases of isolation and identification of the causative agent. Few fungi are considered to be neurotropic because they tend to be located in the CNS after invading the host; however, most cases of meningitis or meningoencephalomyelitis are part of systemic fungal infection such as Candidiasis, Cryptococcosis, Aspergillosis or ZM. This paper documents the discovery of a zigomicotic meningoencephalomyelitis secondary to a head trauma in a prehensile-tailed porcupine (Coendou prehensilis), being a rare disease infrequent in the central nervous system both in human and animals and additionally without previous reports in this specie.

KEY WORDS: Central Nervous System; Fungal Infections; Zygomycosis; Porcupine.

\section{REFERENCIAS BIBLIOGRÁFICAS}

Acha, P. N. \& Szyfres, B. Zoonosis y enfermedades transmisibles comunes al hombre y a los animales. Bacteriosis y Micosis. $3^{\mathrm{a}}$ Ed. Estados Unidos, OPS, 2001. V. 1. pp. 337-9.

Baker, R. D. Leukopenia and therapy in leukemia as factors predisposing to fatal mycoses. Mucormycosis, aspergillosis, and cryptococcosis. Am. J. Clin. Pathol., 37: 358-73, 1962.

Barker, I. K.; Van Dreumel, A. A.; Palmer, N. Mycotic diseases of the gastrointestinal tract. In: Jubb, K.V. F.;
Kennedy, P. C. \& Palmer, N. (Eds.) Pathology of domestic animals. San Diego, Academic Press, 1993. V. 2. pp. 255-7.

Berenguer, B. J. \& Rodríguez, T. J. L. Infecciones profundas por hongos miceliales y dimórficos. Medicine, 7(81):3737-41, 1998.

Carrasco, L.; Bautista, M. J.; de las Mulas, J. M. \& Jensen, H. E. Application of enzyme-immunohistochemistry for the diagnosis of aspergillosis, candidiasis, and zygomycosis in three lovebirds. Avian Dis., 37:923-7, 1993. 
Carrasco, L.; Tarradas, M. C.; Gómez-Villamandos, J. C.; Luque, I.; Arenas, A. \& Méndez, A. Equine pulmonary mycosis due to Aspergillus niger and Rhizopus stolonifer. J. Comp. Pathol., 117:191-9, 1997.

Chakrabarti, A.; Das, A.; Mandal, J.; Shivaprakash, M. R.; George, V. K.; Tarai, B., Rao, P.; Panda, N.; Verma, S. C. \& Sakhuja, V. The rising trend of invasive zygomycosis in patients with uncontrolled diabetes mellitus. Med. Mycol., 44:335-42, 2006.

Chakrabarti A. Epidemiology of central nervous system mycoses. Neurol. India, 55(3):191-7, 2007.

Chandler, F. W. \& Watts, J. C. Pathological diagnosis of fungal infections. Chicago, American Society of Clinical Pathologists Press, 1987. pp. 1-115.

Eisenberg, J. F. \& Redford, K. H. Mammals of the Neotropics, The Central Neotropics. Chicago and London, The University of Chicago Press, 1999. V. III.

Fahey, B. "Coendou prehensilis" Animal Diversity Web, 1999 [Web Document] http:// animaldiversity.ummz.umich.edu/site/accounts/ information/Coendou_prehensilis.html.

García-Rodríguez, J. A. y Picazo, J. J. Compendio de Microbiología Médica. Hongos productores de micosis oportunistas. Madrid, Elsevier, 1999. pp. 424-7.

Harvey, R. G. \& McKeever, P. J. Manual ilustrado de enfermedades de la piel en Perros y Gatos. Grass Ediciones. 2001. p. 132.

IUCN 2008. 2008 IUCN Red List of Threatened Species. www.iucnredlist.org.

Jubb, K.; Kennedy, P. \& Palmer, N. Pathology of Domestics animals. Fungal diseases of skin. Elsevier Saunders, 2007. V.1. pp. 707-8.

Kitt, T. \& Schulz, L. C. Tratado de anatomía patológica general para veterinarios y estudiantes de veterinaria. $2^{\mathrm{a}}$ Ed. Barcelona, Labro S. A. 1985. p. 136.

Kumar, V.; Cotran, R. S. \& Robbins, S. L. Robbins Patología Humana. $7^{\mathrm{a}}$ Ed. Madrid, Elsevier, 2004.

Mayayo, A. E. Diagnóstico histopatológico de las micosis. Rev. Iberoam. Micol., 21:1-9, 2004.

Medleau, L. \& Hnilica, K. A. Dermatología de pequeños animales: Atlas en color y guía terapéutica. Zigomicosis. $2^{\mathrm{a}}$ Ed. Madrid, Elsevier Saunders, 2007. pp. 85-6.

Miller, M. Pathology of Nervous System: Dogs and Cats. En: (Memorias) I Curso Seminario Internacional de Patología Veterinaria. Universidad Nacional de Colombia, Bogota D.C., 13 al 15 de Agosto de 2008: 99, 109-110.

Munday, J. S., Laven, R. A., Orbell, G. M. \& Pandey, S. K. Meningoencephalitis in an adult cow due to Mortierella wolfii. J. Vet. Diagn. Invest., 18:619-22, 2006.

Nowak, R. M. Walker's Mammals of the World. $6^{\text {th }}$ Ed. Baltimore and London, Johns Hopkins University Press, 1999. p. 1936.

Pérez, J. \& Carrasco, L. Diagnóstico histopatológico de micosis en patología veterinaria. Rev. Iberoam. Micol., 17:18-22, 2000.

Ribes, J. A.; Vanover-Sams, C. L. \& Baker, D. J. Zygomycetes in Human Disease. Clin. Microbiol. Rev., 13(2):236-301, 2001.

Riet-Correa, F.; Dantas, A. F.; Azevedo, E. O.; Simões, S. D.; Silva, S. M.; Vilela, R. \& Mendoza, L. Outbreaks of rhinofacial and rhinopharyngeal zygomycosis in sheep in Paraíba, northeastern Brazil. Pesq. Vet. Bras., 28(1):29-35, 2008.

Roberts, M.; Brand, S. \& Maliniak, E. The biology of captive prehensile-tailed porcupines, Coendou prehensilis. J. Mamm., 66(3):476-82, 1985.

Scott, D. W.; Miller, W. H. \& Griffin, C. E. Fungal skin diseases. In: Scott, D. W.; Miller, W. H. \& Griffin, C. E. (Eds.) Small animal dermatology. Philadelphia, W. B. Saunders, 1995. pp. 392-468.

Shelly, S. M. Cutaneous lesions. Vet. Clin. Small Anim., 33:13-4, 2003.

Spraker, T. R. Stress and capture myopathy in artiodactylids In: Fowler, M. E. (ed). Zoo and wildlife medicine: Current therapy. 3th Ed. W. B. Denver, Colorado, Saunders Company, 1993. pp. 481-8.

Sugar, A. M. Mucormycosis. Clin. Infect. Dis., 14:S126-9, 1992.

Sundaram, C.; Mahadevan, A.; Laxmi, V.; Yasha, T. C.; Santosh, V.; Murthy, J. M. K.; Purohit, A. K.; Mohandas, S. \& Shankar, S. K. Cerebral zygomycosis. Mycoses, 48(6):396-407, 2005. 
CIUODERIS-APONTE, K. A. \& OCHOA-AMAYA, J. E. Meningoencefalitis zigomicotica piogranulomatosa en un puercoespin de cola prensil (Coendou Prehensilis). Int. J. Morphol., 27(4):1187-1194, 2009.

Torack, R. M. Fungus infections associated with antibiotic and steroid therapy. Am. J. Med., 22:872-81, 1957.

Vadillo, A.; Piriz, S. \& Mateos, E. M. Manual de microbiología veterinaria. Madrid, Mc-Graw Hill, Interamericana Eds. 2002. pp. 539-43.

Verma, A.; Brozman, B. \& Petito, C. K. Isolated cerebral mucormycosis: Report of a case and review of the literature. J. Neurol. Sci., 240:65-9, 2006.

Wilson, D. E. \& Reeder, D. M. (eds). Mammal Species of the World: A Taxonomic and Geographic Reference. $3^{\text {rd }}$ Ed. Johns Hopkins University Press, 2005, 2142. Disponible en Johns Hopkins University Press, http:// www.press.jhu.edu/
Dirección para correspondencia:

Prof. Julieta Esperanza Ochoa Amaya Escuela de Medicina Veterinaria y Zootecnia

Facultad de Ciencias Agropecuarias y Recursos Naturales Universidad de los Llanos

Villavicencio- Meta

COLOMBIA

E-mail: julietaeochoa@yahoo.es Julieta.ochoa@gmail.com

Recibido : 07-01-2009

Aceptado: 21-08-2009 